\title{
MULTIPLIERS OF BERGMAN SPACES INTO LEBESGUE SPACES
}

\author{
by DANIEL H. LUECKING*
}

(Received 23rd May 1985)

\section{Introduction}

Let $U$ be the open unit disk in the complex plane $\mathbb{C}$ endowed with normalized Lebesgue measure $m$. $L^{p}$ will denote the usual Lebesgue space with respect to $m$, with $0<p<+\infty$. The Bergman space consisting of the analytic functions in $L^{p}$ will be denoted $L_{a}^{p}$. Let $\mu$ be some positive finite Borel measure on $U$. It has been known for some time (see [6] and [9]) what conditions on $\mu$ are equivalent to the estimate: There is a constant $C$ such that

$$
\left(\int|f|^{q} d \mu\right)^{1 / q} \leqq C\left(\int|f|^{p} d m\right)^{1 / p} \quad \text { for all } \quad f \in L_{a}^{p}
$$

provided $0<p \leqq q$. It has been of considerable interest (to the author at least) to obtain a similarly complete result for the remaining cases, namely $0<q<p$. One way the study of (1.1) arises is through consideration of the multiplier problem for Bergman spaces. That is, what conditions on a measurable function $g$ are equivalent to $g L_{a}^{p} \subseteq L^{q}$ ? This reduces, via the closed graph theorem, to the estimate $\left(\int|g f|^{q} d m\right)^{1 / q} \leqq C\left(\int|f|^{p} d m\right)^{1 / p}$, which is (1.1) with $d \mu=|g|^{q} d m$. For $g$ analytic, the problem was solved by K. R. M. Attele in [2] (see also [3]) where the obvious sufficient condition $g \in L_{a}^{r}, 1 / r=1 / q-1 / p$, was shown to be necessary. For a general measure $\mu$, a sufficient condition is easy to come by. It can be shown that $\int|f|^{q} d \mu \leqq C \int|f|^{q} k d m$ where $k(z)$ is a function obtained from $\mu$ by averaging $\mu$ over a hyperbolic neighborhood of $z$ (see the next section). The sufficient condition arises from Holder's inequality and is simply $k \in L^{s}, 1 / s+q / p=1$. In this paper, I show that this condition is necessary.

\section{Background}

For $z, w \in U$ let $\rho(z, w) \equiv|(z-w) /(1-\bar{w} z)|$, the pseudohyperbolic distance between $z$ and $w$. In this metric two points are far apart if the distance between them is nearly 1 .

If $0<\varepsilon<1$ and $a \in U$, let $D_{\varepsilon}(a)=\{z: \rho(z, a)<\varepsilon\}$. Occasionally, when the exact value of $\varepsilon$ is unimportant, I will write $D(a)$ for $D_{\ell}(a)$. $D_{\varepsilon}(a)$ is an actual disk (i.e., in the Euclidean metric) with centre at

$$
\frac{1-\varepsilon^{2}}{1-\varepsilon^{2}|a|^{2}} a \text { and radius } \varepsilon \frac{1-|a|^{2}}{1-\varepsilon^{2}|a|^{2}}
$$

"Supported in part by NSF Grant No. DMS 8201603 and by a J. William Fulbright College of Arts and Sciences full-time research appointment at the University of Arkansas. 
Thus, if $a$ is fixed, $D_{\varepsilon}(a)$ behaves like a disk of radius $\sim \varepsilon$. And if $\varepsilon$ is fixed the radius behaves like $1-|a|^{2}$. Its normalized area is

$$
m\left(D_{\varepsilon}(a)\right)=\varepsilon^{2}\left(\frac{1-|a|^{2}}{1-\varepsilon^{2}|a|^{2}}\right)^{2}
$$

Because $|f|^{q}$ is subharmonic for $f \in L_{a}^{q}$, it follows that $\int_{D(a)}|f|^{q} d m / m(D(a))$ exceeds the value of $|f|^{q}$ at the centre of $D(a)$. If $\varepsilon$ is fixed, the distance from $a$ to the centre of $D_{\varepsilon}(a)$ is at most $\varepsilon|a|$ times the radius of $D_{\varepsilon}(a)$. By subharmonicity again, there is a constant $C$ depending only on $\varepsilon$ such that

$$
C \int_{D(a)}|f|^{a} d m / m(D(a)) \geqq|f(a)|^{q}
$$

(This inequality is also valid for harmonic functions, except that $C$ will also depend on $q$ if $q<1$. Indeed, the proof of Lemma 2, page 152 of [5], shows that one only needs $|f|$ to be subharmonic.) Using (2.1) to estimate $|f|^{q}$ in $\int|f|^{q} d \mu$ and applying Fubini's theorem, one obtains

$$
\int|f|^{q} d \mu \leqq C \int|f(z)|^{q} \int_{D(z)} \frac{1}{m(D(w))} d \mu(w) d m(z) .
$$

It is easy to verify that if $w \in D(z)$ then

$$
\frac{m(D(z))}{m(D(w))} \leqq C
$$

with $C$ depending on $\varepsilon$. Thus, putting $k(z)=\mu(D(z)) / m(D(z))$, one gets

$$
\int|f|^{q} d \mu \leqq C \int|f|^{q} k d m
$$

An immediate result is the following:

If $k$ belongs to $L^{s}$ for $s=p /(p-q)$, then

$$
\left(\int|f|^{q} d \mu\right)^{1 / q} \leqq C\left(\int|f|^{p} d m\right)^{1 / p} \quad \text { for all } f \in L_{a}^{p} .
$$

The constant $C$ depends only on $\varepsilon, q$, and the value of $\int k^{s} d m$. The main theorem is the converse of (2.3):

Theorem. Let $\mu$ be a positive measure on $U$ and let $k(z)=\mu(D(z)) / m(D(z))$ where $D(z)$ $=D_{\varepsilon}(z)$ for some convenient $\varepsilon \in(0,1)$. Let $0<q<p$. Then a necessary and sufficient condition for there to exist a constant $C$ satisfying

$$
\left(\int|f|^{q} d \mu\right)^{1 / q} \leqq C\left(\int|f|^{p} d m\right)^{1 / p}
$$

for all $f \in L_{a}^{p}$ is that $k$ belong to $L^{s}$, where $1 / s+q / p=1$. 
This will be proved in Section 3. The remainder of this section is devoted to showing that the condition $k \in L^{s}$ is independent of the choice of $\varepsilon \in(0,1)$.

Lemma. Let $0<\delta<\varepsilon<1$ and let $k_{\varepsilon}(z)=\mu\left(D_{\varepsilon}(z)\right) / m\left(D_{\varepsilon}(z)\right)$ with $k_{\delta}$ defined similarly. If $s \geqq 1$, then $k_{\varepsilon} \in L^{s}$ if and only if $k_{\delta} \in L^{s}$.

Proof. Clearly $k_{\delta}(z) \leqq k_{\varepsilon}(z)\left[m\left(D_{\varepsilon}(z)\right) / m\left(D_{\delta}(z)\right)\right]$ and the formula for the area of pseudohyperbolic disks shows that $m\left(D_{z}(z)\right) / m\left(D_{\delta}(z)\right)$ is a bounded function of $z$. Thus $k_{\varepsilon} \in L^{s}$ implies $k_{\delta} \in L^{s}$. Now suppose $k_{\delta} \in L^{s}$ and let $\phi(z)=\int_{D_{\varepsilon}(z)} k_{\delta} d m / m\left(D_{\varepsilon}(z)\right)$. It is an easy exercise with Fubini's theorem to show that if $k_{\delta}$ is in $L^{1}$ then so is $\phi$ and it is even clearer that if $k_{\delta}$ is bounded so is $\phi$. By any of a variety of interpolation theorems it follows that if $k_{\delta} \in L^{s}$, then also $\phi \in L, 1 \leqq s<\infty$. Finally, the following estimates show that $\phi$ dominates $k_{\varepsilon}$ :

$$
\begin{aligned}
\int_{D_{\varepsilon}(z)} k_{\delta} d m & =\int_{D_{\varepsilon}(z)} \int_{D_{\delta}(w)} d \mu(t) / m\left(D_{\delta}(w)\right) d m(w) \\
& =\iint \chi_{D_{\varepsilon}(z)}(w) \chi_{D_{\delta}(w)}(t) m\left(D_{\delta}(w)\right)^{-1} d m(w) d \mu(t) \\
& \geqq \mathcal{c} \iint \frac{m\left(D_{\varepsilon}(z) \cap D_{\delta}(t)\right)}{m\left(D_{\delta}(t)\right)} d \mu(t)
\end{aligned}
$$

It is clear that the integrand exceeds $1 / 3$ when $t$ lies in $D_{\varepsilon}(z)$, so

$$
\int_{D_{\varepsilon}(z)} k_{\delta} d m \geqq c \mu\left(D_{\varepsilon}(z)\right) \text {. }
$$

\section{Interpolating sequences}

In order to obtain an integrability condition on $k$ from an inequality like (1.1), it has to be shown $|f|^{q}$ can be made "sufficiently arbitrary". Think of a discrete version of $k$ obtained by decomposing the disk into hyperbolically "equal"-sized pieces $\left\{D_{i}\right\}$ as in [4] and putting $k$ on each of these pieces equal to the average of $\mu$ on that piece. It is not hard to show that the condition on $\mu\left(k \in L^{s}\right)$ is equivalent to

$$
\sum\left(\frac{\mu\left(D_{i}\right)}{m\left(D_{i}\right)}\right)^{s} m\left(D_{i}\right)<+\infty .
$$

Then $\int|f|^{a} d \mu$ ought to be roughly $\sum \int_{D_{i}}|f|^{a} d m \mu\left(D_{i}\right) / m\left(D_{i}\right)$, so we would like to make $\int_{D_{i}}|f|^{q} d m / m\left(D_{i}\right)$ dominate an arbitrary sequence in the weighted $\beta^{\prime}$ space with weights $m\left(D_{i}\right), s^{\prime}=p / q$. This can be done by making sure each $D_{i}$ contains a point $a_{i}$ so that $\left\{a_{i}\right\}$ is an interpolation sequence for $L_{a}^{p}$. The rest of the proof of the main theorem consists of making this intuition precise.

Definition. A sequence $\left\{a_{i}\right\}$ in $U$ is said to be separated if there exists a $\delta>0$ such that $\rho\left(a_{i}, a_{j}\right)>\delta$ when $i \neq j$. A separated sequence $\left\{a_{i}\right\}$ is called an interpolation sequence 
for $L_{a}^{p}$ if whenever $\left\{c_{i}\right\}$ is a sequence of complex numbers such that $\sum\left|c_{i}\right|^{p}\left(1-\left|a_{i}\right|^{2}\right)^{2}<$ $+\infty$, then there exists $f \in L_{a}^{p}$ satisfying $f\left(a_{i}\right)=c_{i}$.

Because $\left|f\left(a_{i}\right)\right|^{p} m\left(D_{\delta}\left(a_{i}\right)\right) \leqq C \int_{D_{\delta}\left(a_{i}\right)}|f|^{p} d m$, it follows that if $\left\{a_{i}\right\}$ is $2 \delta$-separated, then the operator $R f=\left\{f\left(a_{i}\right)\right\}$ is a bounded map of $L_{a}^{p}$ into the weighted sequence space $I^{p}\left\{\left(1-\left|a_{i}\right|^{2}\right)^{2}\right\}$. A sequence $\left\{a_{i}\right\}$ is an interpolation sequence if $R$ is onto. It follows from the open mapping theorem that a constant $M$ may be associated with any given interpolation sequence $\left\{a_{i}\right\}$ such that any $\left\{c_{i}\right\} \in l^{p}\left\{\left(1-\left|a_{i}\right|^{2}\right)^{2}\right\}$ with $\sum\left|c_{i}\right|^{p}\left(1-\left|a_{i}\right|^{2}\right)^{2} \leqq 1$ is the image under $R$ of a function $f \in L_{a}^{p}$ with $\left(\int|f|^{p} d m\right)^{1 / p} \leqq M$. This $M$ will be referred to as the interpolation constant of $\left\{a_{i}\right\}$.

It is a result of Eric Amar [1] (but see also [10]) that if $\left\{a_{i}\right\}$ is a separated sequence, then it is the union of finitely many interpolation sequences. Specifically, the following was shown.

Theorem. (E. Amar) If $\left\{b_{i}\right\}$ is a $\delta$-separated sequence, then $\left\{b_{i}\right\}$ is the union of $N=N(\delta, \eta) \eta$-separated sequence, and if $\eta$ is near enough to 1 then each $\eta$-separated sequence is an interpolation sequence for $L_{a}^{p}$. The size of $\eta$ will depend on $p$ and the interpolation constant $M$ will depend only on $\eta$ and $p$.

Now fix $\eta>\frac{1}{2}$ once and for all, so near to 1 that any $\eta$-separated sequence is an interpolation sequence. This fixes an interpolation constant $M$. Let $\delta \in(0,1)$ be a small number; its actual size will be specified later and will depend only on $\eta, M$ and the constant $C$ in the estimate (2.4) of the main theorem. Construct a $\delta / 2$-lattice, that is, a $\delta / 2$-separated sequence $\left\{b_{i}\right\}$ such that the disks $\left\{D_{\delta / 2}\left(b_{i}\right)\right\}$ cover $U$. Here is a simple construction: let $b_{1}=0$, and once $b_{1}$ through $b_{n-1}$ are obtained, pick $b_{n} \notin \bigcup_{1}^{n-1} D_{\delta / 2}\left(b_{i}\right)$ which minimizes $\left|b_{n}\right|$. Clearly $\left\{b_{i}\right\}$ will be $\delta / 2$-separated. If $z_{0} \notin \cup D_{\delta / 2}\left(b_{i}\right)$ then all $b_{i}$ lie in $\left\{z:|z|<\left|z_{0}\right|\right\}$ or else $z_{0}$ was needlessly overlooked in the selection. A contradiction has been reached in that infinitely many disjoint $D_{\delta / 4}\left(b_{i}\right)$ have their centres in $|z|<\left|z_{0}\right|$. The proof of the following lemma is quite similar to arguments used in [7] and [8].

Lemma. There is a constant $A$ depending only on $q$ and $\eta$ such that if $\left\{a_{i}\right\}$ is an $\eta$ separated sequence and $\delta$ is sufficiently small, then for every $f \in L_{a}^{p}$

$$
\sum \int_{D_{\delta}\left(a_{i}\right)}\left|f(z)-f\left(a_{i}\right)\right|^{q} d \mu(z) \leqq A \delta^{q}\|f\|_{L p}^{q}\left(\sum \mu\left(D_{\delta}\left(a_{i}\right)\right)^{s} m\left(D_{i}\right)^{1-s}\right)^{1 / s}
$$

where $D_{i}=D_{\eta / 2}\left(a_{i}\right)$.

Proof. It is clear by normal families and scaling that if $|z|<\delta<\eta / 4$ and $D=\{z:|z|<\eta / 2\}$, then

$$
\left|\frac{f(z)-f(0)}{z}\right|^{q} \leqq C \int_{D}|f|^{q} d m
$$

where $C$ depends only on $q$, if that. Thus $|f(z)-f(0)|^{q} \leqq C \delta^{q} \int_{D}|f|^{q} d m$. The change of 
variables $z \rightarrow\left(z-a_{i}\right) /\left(1-\bar{a}_{i} z\right)$ gives

$$
\begin{aligned}
\left|f(z)-f\left(a_{i}\right)\right|^{q} & \leqq C \delta^{q} \int_{D_{i}}|f|^{q} \frac{\left(1-\left|a_{i}\right|^{2}\right)^{2}}{\left|1-\bar{a}_{i} z\right|^{4}} d m \\
& \leqq A \delta^{q} \int_{D_{i}}|f|^{q} d m / m\left(D_{i}\right)
\end{aligned}
$$

where the estimate $\left(1-\left|a_{i}\right|^{2}\right)^{2} /\left|1-\bar{a}_{i} z\right|^{4} \leqq$ constant $m\left(D_{i}\right)^{-1}$ has been used for $z \in D_{i}$. The constant depends only on $\eta$. Integrating (3.2) with respect to $\mu$ over $D_{\delta}\left(a_{i}\right)$, and summing, one sees that the left-hand side of (3.1) is at most

$$
\begin{gathered}
A \delta^{q} \sum \int_{D_{i}}|f|^{q} d m \mu\left(D_{\delta}\left(a_{i}\right)\right) m\left(D_{i}\right)^{-1} \leqq A \delta^{q} \sum\left(\int_{D_{i}}|f|^{p} d m\right)^{q / p} \mu\left(D_{\delta}\left(a_{i}\right)\right) m\left(D_{i}\right)^{1 / s-1} \\
\leqq A \delta^{q}\left(\sum \int_{D_{i}}|f|^{p} d m\right)^{q / p}\left(\sum \mu\left(D_{\delta}\left(a_{i}\right)\right)^{s} m(D)^{1-s}\right)^{1 / s}
\end{gathered}
$$

(recall $s$ is just the conjugate exponent of $p / q$ ).

Since the $D_{i}$ are disjoint, the expression in the first parentheses is at most $\|f\|_{L^{p}}^{p}$.

The proof of the main theorem may now be completed. To this end let $\mu$ be a measure satisfying the integral inequality (2.4) of the theorem. If we replace $\mu$ with $\chi_{\{|z|<r\}} \mu$, then (2.4) is still valid with the same constant. If we show that the estimate on $\|k\|_{L^{\text {s }}}$ is independent of $r$, we may let $r \rightarrow 1$ to obtain the theorem. Thus, without any loss of generality, $\mu$ is compactly supported in $U$ and all of the sums below involving $\mu$ are finite. Let $\left\{b_{i}\right\}$ be the $\delta / 2$-lattice constructed earlier and let $\left\{a_{k}\right\}$ be one of the $N((\delta / 2), \eta)$ $\eta$-separated sequences whose union is $\left\{b_{i}\right\}$. Let $M$ be its interpolation constant. From the lemma, if $f \in L_{a}^{p},\|f\|_{L^{p}} \leqq M$, and $q \leqq 1$ then

$$
\begin{aligned}
\sum \int_{D_{\delta}\left(a_{k}\right)}|f|^{q} d \mu & \geqq \sum \int_{D_{\delta}\left(a_{k}\right)}\left|f\left(a_{k}\right)\right|^{q} d \mu-\sum \int_{D_{\delta}\left(a_{k}\right)}\left|f-f\left(a_{k}\right)\right|^{q} d \mu \\
& \geqq \sum\left|f\left(a_{k}\right)\right|^{q} \mu\left(D_{\delta}\left(a_{k}\right)\right)-A \delta^{q} M^{q}\left(\sum \mu\left(D_{\delta}\left(a_{k}\right)\right) m\left(D_{k}\right)^{1-s}\right)^{1 / s}
\end{aligned}
$$

where $D_{k}=D_{\eta / 2}\left(a_{k}\right)$ as in the lemma. Since $f\left(a_{k}\right)$ may assume the values of any sequence $\left\{c_{k}\right\}$ with $\left.\sum\left|c_{k}\right|^{p}\left(1-\left|a_{k}\right|\right)^{2}\right)^{2}=1$, the sum $\sum\left|f\left(a_{k}\right)\right|{ }^{q} \mu\left(D_{\delta}\left(a_{k}\right)\right)$ may assume the value $\left(\sum \mu\left(D_{d}\left(a_{k}\right)\right)^{s}\left(1-\left|a_{k}\right|^{2}\right)^{2(1-s)}\right)^{1 / s} \geqq \beta\left(\sum \mu\left(D_{\delta}\left(a_{k}\right)\right) m\left(D_{k}\right)^{1-s}\right)^{1 / s}$. Here $\beta$ depends only on $\eta$. Thus we have

$$
\begin{aligned}
C^{q} M^{q} & \geqq \sum \int_{D_{\delta}\left(a_{k}\right)}|f|^{q} d \mu \\
& \geqq\left(\beta-A \delta^{q} M^{q}\right)\left(\sum \mu\left(D_{\delta}\left(a_{k}\right)\right)^{s} m\left(D_{k}\right)^{1-q}\right)^{1 / s} .
\end{aligned}
$$


We now choose $\delta^{q}=\beta\left(2 A M^{q}\right)$, and sum over the $N$ sequences $\left\{a_{k}\right\}$ to get

$$
\left(\sum \mu\left(D_{\delta}\left(b_{i}\right)\right)^{s} m\left(D_{i}\right)^{1-s}\right)^{1 / s}<2 N C^{q} M^{q} / \beta
$$

where $D_{i}=D_{\eta / 2}\left(b_{i}\right)$. It remains to be shown that (3.3) implies $k \in L^{s}$. Set $\varepsilon=\delta / 2$ and define $k(z)=\mu\left(D_{\varepsilon}(z)\right) / m\left(D_{\varepsilon}(z)\right)$. If $z \in D_{\varepsilon}\left(b_{i}\right)$, then $D_{\varepsilon}(z) \subseteq D_{\delta}\left(b_{i}\right)$ and so $k(z) \leqq \mu\left(D_{\delta}\left(b_{i}\right)\right) / m\left(D_{\varepsilon}(z)\right) \leqq$ constant $\mu\left(D_{\delta}\left(b_{i}\right)\right) / m\left(D_{i}\right)$. Thus $\sum \int_{D_{\varepsilon}\left(b_{i}\right)} k^{s} d m \leqq$ constant $\sum \mu\left(D_{\delta}\left(b_{i}\right)\right)^{s} m\left(D_{i}\right)^{-s} m\left(D_{e}\left(b_{i}\right)\right) \leqq$ constant $\sum \mu\left(D_{\delta}\left(b_{i}\right)\right)^{s} m\left(D_{i}\right)^{1-s}<$ constant by (3.3). Since the disks $D_{e}\left(b_{i}\right)$ cover $U$ we get $\int_{U} k^{s} d m \leqq$ constant, where the constant depends only on $N, C, M, q, \beta, \eta$, and $\delta$. That is, ultimately only on $C, q$, and $p$. If $q>1$ only minor changes are needed in (3.3). The proof is completed.

\section{Remarks}

It should come as no surprise that the theorem remains valid, mutatis mutandis, when the disk is replaced by the unit ball in $\mathbb{C}^{n}$, Lebesgue measure $m$ is replaced by a weighted measure $m_{\alpha}\left(1-|z|^{2}\right)^{\alpha} m$, and analytic functions are replaced by pluriharmonic functions. In fact, thanks to Richard Rochberg's extension [10] of Eric Amar's result on interpolation sequences, there is a formulation, left to the reader, of the theorem that is valid in weighted Bergman spaces on bounded symmetric domains in $\mathbb{C}^{n}$.

Acknowledgement. Thanks are due to the Department of Mathematics at the University of North Carolina at Chapel Hill for their hospitality while the research for this paper was done.

\section{REFERENCES}

1. E. Amar, Suites d'interpolation pour les class de Bergman de la boule et du polydisque de $\mathbb{C}^{n}$, Canad. J. Math. 30 (1978), 711-737. 319.

2. K. R. M. Atrele, Analytic multipliers of Bergman spaces, Mich. Math. J. 31 (1984), 307-

3. S. AxLER, Zero multipliers of Berman spaces, Canad. Math. Bull. 28 (1985), 237-242.

4. R. CoIfman and R. Rochberg, Representation theorems for holomorphic and harmonic functions, Astérisque 77 (1980), 11-65.

5. C. Fefferman and E. Stein, $H^{p}$ spaces of severable variables, Acta Math. 129 (1972), 128-193.

6. W. W. Hastings, A Carleson measure theorem for Bergman spaces, Proc. Amer. Math. Soc. 52 (1975), 237-241.

7. D. H. Luecking, Forward and reverse Carleson inequalities for functions in the Bergman spaces and their derivatives, Amer. J. Math. 107 (1985), 85-111.

8. D. H. LUECKING, Representation and duality in weighted spaces of analytic functions, Indiana Univ. Math. J. 34 (1985), 319-336. 
9. V. L. OleiniK and B. S. Pavlov, Embedding theorems for weighted classes of harmonic and analytic functions, J. Soviet Math. 2 (1974), 135-142 (a translation of Zap. Nauch. Sem. LOMI Steklov 22 (1971)).

10. R. RochBerG, Interpolation by functions in the Bergman spaces, Mich. Math. J. 29 (1982), 229-236.

University of Arkansas

FAyetTEVILLE

Arkansas 72701 